Cas clinique

\title{
La glomérulonéphrite fibrillaire non amyloïde : une cause rare de syndrome néphrotique
}

\author{
Fibrillary nonamyloid glomerulonephritis: A rare etiology of nephrotic syndrome \\ Stéphanie Grosch *, Lionel Van Overmeire, Jean-Marie Krzesinski, Christophe Bovy \\ Service de néphrologie du CHU de Liège, université de Liège, domaine universitaire du Sart-Tilman, B35, 4000 Liège, Belgique
}

\section{N F O A R T I C L E}

\section{Historique de l'article :}

Reçu le 16 novembre 2010

Received in revised form 3 March 2011

Accepté le 5 mars 2011

\section{Mots clés :}

Glomérulonéphrite fibrillaire

Glomérulopathies à dépôts non amyloïdes

Syndrome néphrotique

\section{Keywords:}

Fibrillary glomerulonephritis

Glomerular deposition disease

Nephrotic syndrome

\begin{abstract}
R É S U M É
La glomérulonéphrite fibrillaire non amyloïde est une pathologie glomérulaire à dépôts fibrillaires, non amyloïdes, composés principalement d'immunoglobulines $G$ polyclonales. Il s'agit d'une glomérulopathie idiopathique responsable de protéinurie sévère, souvent néphrotique et d'insuffisance rénale arrivant à un stade terminal dans $40 \%$ des cas à cinq ans. Elle peut prendre différents aspects anatomopathologiques déterminants en terme de pronostic rénal. La négativité des dépôts en coloration rouge Congo et l'épaisseur des fibrilles en microscopie électronique permettent le diagnostic différentiel avec les dépôts amyloïdes. Il n'y a pas de traitement efficace. Après transplantation rénale, la récidive est fréquente avec un pronostic rénal cependant moins péjoratif.
\end{abstract}

(c) 2011 Association Société de néphrologie. Publié par Elsevier Masson SAS. Tous droits réservés.

A B S T R A C T

The fibrillary nonamyloïd glomerulonephritis is a glomerulopathy with fibrillar, nonamyloid deposits of predominantly polyclonal immunoglobulin G. It is an idiopathic glomerulopathy responsible for heavy proteinuria, generally in the nephrotic range, and renal failure up to end stage in $40 \%$ of the cases after five years. The histologic pattern is variable, correlating with renal prognosis. The Congo red-negativity of the deposits and the size of the fibrils on electron microscopy make the differential diagnosis with amyloid deposits. There is no specific efficient therapy. Recurrence in the transplant is frequent, but with better renal prognosis.

() 2011 Association Société de néphrologie. Published by Elsevier Masson SAS. All rights reserved.

\section{Abréviations}

RCUH rectocolite ulcérohémorragique

Ig immunoglobuline

VIH virus de l'immunodéficience humaine

PAS acide périodique de Schiff (periodic acid Schiff)

GIST tumeur stromale gastro-intestinale

GNEM glomérulonéphrite extramembraneuse

GNFNA glomérulonéphrite fibrillaire non amyloïde

AA amyloïdose AA (amyloïde A)

$\mathrm{AL} \quad$ amyloïdose $\mathrm{AL}$ (chaînes légères)

GPIT glomérulopathie immunotactoïde

\section{Cas clinique}

Nous rapportons le cas d'une patiente de 59 ans, caucasienne, qui se présente à la consultation de néphrologie en mars 2010 pour la prise en charge d'un syndrome néphrotique diagnostiqué en Guadeloupe, après plusieurs mois de plaintes, et traité empiriquement, sans diagnostic anatomique, par corticoïdes (prednisolone $60 \mathrm{mg}$ par jour) et antagonistes des récepteurs de l'angiotensine II (losartan $100 \mathrm{mg}$ ), les inhibiteurs de l'enzyme de conversion n'ayant pas été supportés (toux irritative).

Dans les antécédents de la patiente, on note une RCUH actuellement non traitée, une hypercholestérolémie traitée par statine (rosuvastatine $20 \mathrm{mg}$ ) et un tabagisme actif ( 25 cigarettes par jour depuis l'âge de 18 ans).

À l'anamnèse, la patiente décrit des lipothymies dues à une hypotension artérielle, la présence d'œdèmes des membres inférieurs avec une prise de poids de $10 \mathrm{~kg}$ sur les derniers mois et des rectorragies.

\footnotetext{
* Auteur correspondant.

Adresse e-mail : sgrosch@chu.ulg.ac.be (S. Grosch).
} 
L'examen clinique est normal hormis la présence d'œdèmes des membres inférieurs, remontant jusqu'à la racine des cuisses, et prenant le godet. La pression artérielle mesurée couchée et assise est de $110 / 70 \mathrm{mmHg}$.

La biologie sanguine montre une fonction rénale normale (créatinine : 0,8 $\mathrm{mg} / \mathrm{dL}$, MDRD : $>60 \mathrm{ml} / \mathrm{min}$ pour $1,73 \mathrm{~m}^{2}$ ), une hypoprotéinémie $(48 \mathrm{~g} / \mathrm{L})$ avec une hypoalbuminémie $(20 \mathrm{~g} / \mathrm{L})$, une hypercholestérolémie (cholestérol total : 4,65 g/L). Le bilan urinaire met en évidence une protéinurie sévère $(30 \mathrm{~g} / \mathrm{g}$ de créatinine urinaire dont $55 \%$ d'albumine) sans hématurie. Au bilan immunologique, l'électrophorèse des protéines sériques est normale mais avec une diminution des immunoglobulines (IgG), les fractions du complément sont normales. Il n'y a pas de cryoglobulinémie et le bilan immunitaire est négatif (anticorps antineutrophiles, antinucléaires, antimembranes basales glomérulaires et tubulaires). Les sérologies virales reviennent négatives pour le virus de l'immunodéficience humaine (VIH), l'hépatite $B$ et $C$.

Une biopsie rénale est alors réalisée. Environ 80 \% des glomérules présentent des dépôts amorphes, épaississant les membranes basales glomérulaires, non relevés par la coloration argentique, ni par la PAS. Certains lobules glomérulaires sont totalement sclérosés, alors que d'autres apparaissent normaux. Il n'y a pas de composante proliférative. Il n'y a pas d'atrophie tubulaire ou de fibrose interstitielle significative (Fig. 1). Les immunofixations pour IgG, IgM, C1q, et C3d sont positives au niveau des dépôts. L'IgG et le C3d sont également positifs, de manière focale, le long des membranes basales glomérulaires (Fig. 1). La coloration rouge Congo est négative. Les chaînes légères sont positives au niveau des dépôts sans prédominance kappa ou lambda. Dans l'attente des résultats de la microscopie électronique, au vu des dépôts le long des membranes basales, probablement dans la région sous-épithéliale, un diagnostic de glomérulonéphrite extramembraneuse (GNEM) compliquée d'une hyalinose segmentaire et focale secondaire est évoqué, quoique très atypique.

Dans ce contexte et étant donné la présence de rectorragies, une évaluation digestive met en évidence, outre des polypes sigmoïdiens, une tumeur gastrique sous-muqueuse avec un bilan d'extension négatif. L'exérèse de cette tumeur est réalisée en mai 2010 et l'anatomopathologie pose le diagnostic de tumeur stromale gastro-intestinale (GIST). Au vu de la faible propension mitotique de ce type de tumeur et une exérèse en marges saines, aucun traitement adjuvant n'est entrepris et la patiente est considérée comme guérie, sous réserve d'un suivi oncologique régulier. L'exérèse de cette tumeur reste, cependant, sans effet sur le syndrome néphrotique.

Dès le diagnostic partiel de GNEM, un traitement par cyclophosphamide (50 mg deux fois par jour per os) et corticoïdes ( $1 \mathrm{mg} / \mathrm{kg}$ par jour per os à doses dégressives) est prescrit sans amélioration de la protéinurie. Après six semaines de traitement, la patiente présente un épisode sévère d'érysipèle et le cyclophosphamide est alors arrêté et remplacé par de la ciclosporine (100 mg deux fois par jour). Des anti-inflammatoires nonstéroïdiens sont introduits, à visée hémodynamique, mais rapidement arrêtés en raison d'une détérioration, heureusement réversible de la fonction rénale.

Enfin, la microscopie électronique montre la présence d'un matériel amorphe laminant la membrane basale glomérulaire, principalement sur le versant sous-épithélial, ainsi qu'un effacement avec fusion des pieds podocytaires. À plus fort grossissement, les dépôts prennent nettement un aspect fibrillaire, organisé de manière anarchique (Fig. 1). Le diagnostic de glomérulonéphrite fibrillaire non amyloïde (GNFNA) est donc alors retenu.

Devant l'inefficacité thérapeutique de la ciclosporine et la détérioration progressive de la fonction rénale (créatinine : 2,3 mg/
dL, MDRD : $21 \mathrm{ml} / \mathrm{min}$ pour $1,73 \mathrm{~m}^{2}$ ), ce traitement vient d'être arrêté. Au vu des données de la littérature, aucun autre traitement immunosuppresseur n'a été prescrit.

\section{La glomérulonéphrite fibrillaire}

"Les glomérulopathies à dépôts organisés " sont un groupe d'atteintes rénales dans lesquelles des dépôts de microfibrilles ou de microtubules sont retrouvés au niveau du mésangium et des membranes basales glomérulaires. Les microfibrilles n'ont pas de lumière et sont arrangées de manière anarchique. Elles peuvent être dérivées de protéines amyloïdes, d'immunoglobulines ou de protéines de la matrice extracellulaire. Au contraire, les microtubules ont une lumière, sont organisés en structures parallèles et composés uniquement d'immunoglobulines [1,2] (Fig. 2).

La GNFNA est une glomérulopathie avec accumulation de dépôts fibrillaires dans le mésangium et les anses capillaires glomérulaires [1]. Ces dépôts ressemblent à ceux retrouvés dans les amyloïdoses mais sont plus épais en microscopie électronique et sont négatifs au rouge Congo [3,4].

La glomérulopathie immunotactoïde (GPIT) est une entité apparentée avec des dépôts microtubulaires, de plus grande taille (>30 nm), dans les mêmes localisations [3]. Dans la littérature, le débat reste ouvert quant à savoir s' il s'agit de deux entités distinctes avec des tableaux cliniques et physiopathologiques différents incluant des pathologies systémiques (lupus érythémateux, cryoglobulinémie) ou lymphoprolifératives [5,6] versus une seule entité regroupant les glomérulopathies à dépôts non amyloïdes (rouge Congo négatif) d'aspects et de tailles différents chez des patients chez qui une maladie systémique ou lymphoproliférative a été exclue [7-9].

La GNFNA représente 0,5 à $1 \%$ des biopsies de reins natifs $[1,5,10]$. La microscopie optique peut montrer différents aspects histologiques : glomérulonéphrite membranoproliférative (44\%), prolifération ou sclérose mésangiale $(21 \%)$, prolifération diffuse avec prolifération endocapillaire (15\%), glomérulosclérose (13\%) et glomérulonéphrite extramembraneuse (7\%). Il existe également de rares cas où les glomérules apparaissent normaux, en microscopie optique, mimant une glomérulonéphrite à lésions minimes. $[1,4,5,10]$.

Les glomérules sont le siège de dépôts amorphes, acellulaires au niveau du mésangium et des parois des capillaires périphériques de la touffe glomérulaire. Tout comme les dépôts amyloïdes, ces dépôts sont faiblement colorés par le PAS et négatifs en coloration argentique (Jones ou Méthénamine d'argent) mais contrairement aux dépôts amyloïdes, ils sont également négatifs en rouge Congo ou à la thioflavine $\mathrm{T}[4,11]$.

La présence de croissants n'est pas rare (de $20 \%$ à $30 \%$ selon les études) et est souvent associée à une prolifération endocapillaire diffuse $[4,10,11]$.

La fibrose interstitielle et l'atrophie tubulaire sont secondaires et corrélées à la sévérité de l'atteinte glomérulaire. Les membranes basales tubulaires sont rarement le siège de dépôts (5\%). De même, les vaisseaux ne montrent pas de lésions spécifiques $[4,10,11]$.

L'immunofluorescence montre en général, des dépôts polyclonaux d'IgG et de C3. Il s'agit principalement d'IgG4 et moins souvent d'IgG1. Il existe seulement un faible pourcentage de cas avec des dépôts monoclonaux ou oligoclonaux. Ces dépôts se situent au niveau du mésangium et le long des membranes basales glomérulaires avec un aspect granulaire ou pseudo-linéaire. Les immunofixations pour les chaînes légères kappa et lambda peuvent être positives sans prépondérance d'un type de chaîne par rapport à l'autre. Il existe des dépôts faibles d'IgA, d'IgM et de C1q dans 30 à $50 \%$ des cas $[1,5,6,10]$. 

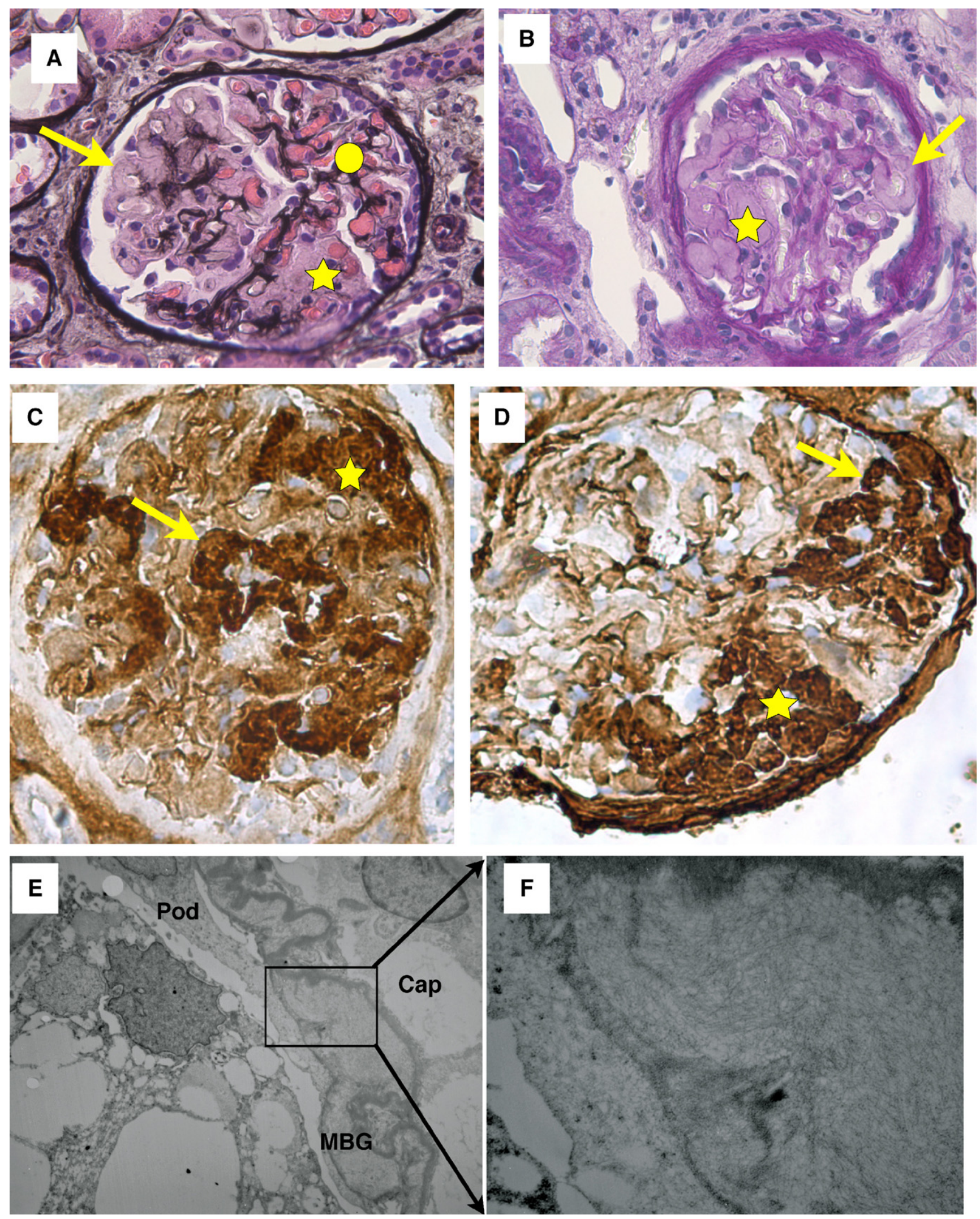

Fig. 1. A. Coloration de Jones, $40 \times$. B. PAS, $40 \times$ : glomérule présentant les différents types de lésions identifiées. $(\rightarrow)$ : épaississement des membranes basales glomérulaires mimant une GNEM de type IV ; $\left({ }^{*}\right):$ sclérose lobulaire ; $(0)$ lobule normal. C. Immunoperoxydase pour IgG, $40 \times$. D. Immunoperoxydase pour C3d, $40 \times:$ positivité de l'IgG et du C3d le long des membranes basales $(\rightarrow)$ et dans les zones sclérosées $\left({ }^{*}\right)$. E. Microscopie électronique, $2156 \times$ : importants dépôts peu denses sur le versant épithélial de la membrane basale glomérulaire (MBG) (CAP : lumière capillaire ; POD : podocyte). F. Microscopie électronique, $10000 \times$ : à fort grossissement, structure finement fibrillaire des dépôts ayant une organisation anarchique. 


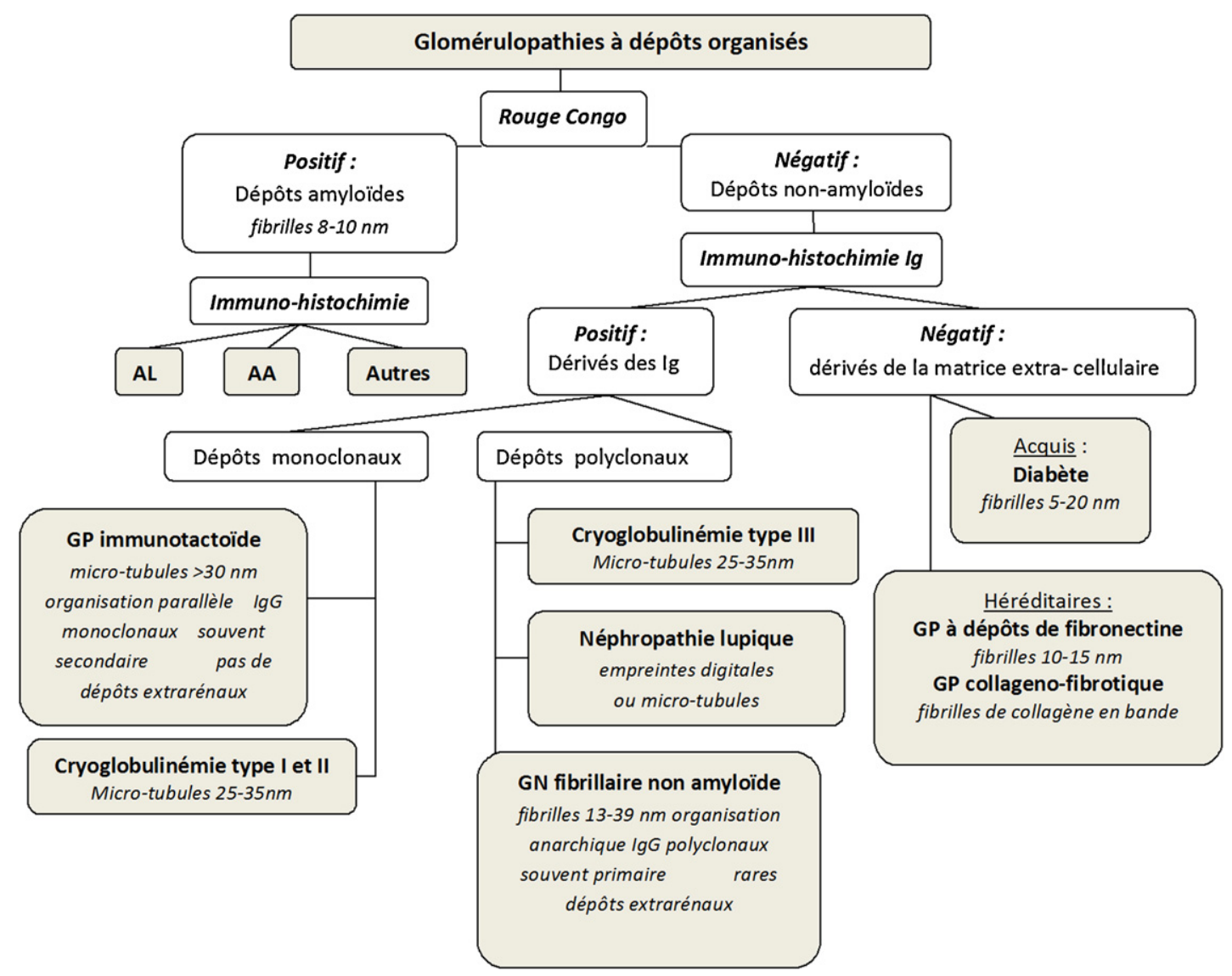

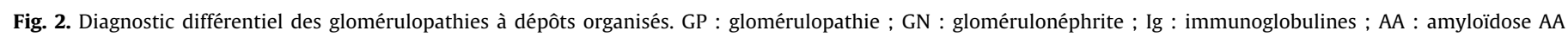
(amyloïde A) ; AL : amyloïdose AL (chaînes légères).

La microscopie électronique permet la confirmation diagnostique de la GNFNA. Elle montre des fibrilles non ramifiées, disposées de manière anarchique, ressemblant aux fibrilles d'amyloïdes mais plus épaisses que celles-ci (13 à $39 \mathrm{~nm}$ versus $10 \mathrm{~nm}$ ). Au vu de l'existence de microfibrilles non amylö̈des de plus petite taille, il est cependant conseillé de tenir compte des données d'immunofluorescence et de la coloration rouge Congo pour aider au diagnostic différentiel. Ces fibrilles sont retrouvées dans tous les compartiments glomérulaires : au niveau du mésangium, au sein de la membrane basale, au niveau sousendothélial ou sous-épithélial, mais rarement au niveau des membranes basales tubulaires $[1,4,10]$.

L'étiologie des GNFNA est inconnue. Contrairement aux GPIT, elle est rarement associée à des maladies lymphoprolifératives et est considérée, le plus souvent, comme une atteinte primitive. Le bilan immunologique et les sérologies sont donc habituellement négatifs $[1,10,11]$ mais il existe quelques cas rapportés de GNFNA concomitantes à une infection à hépatite $C[12]$. Les dépôts sont essentiellement rénaux, mais il existe des cas rapportés dans la littérature de dépôts extra-rénaux, au niveau cardiaque et pulmonaire $[13,14]$. On pense que la formation des fibrilles est secondaire au dépôt de protéines anormales, polyclonales ou monoclonales ou de complexes immuns qui sont, dans un deuxième temps, capables de fabriquer ces structures fibrillaires. La découverte de "cryo-précipitats " fibrillaires (complexes immunoglobuline-fibronectine) dans le sérum de patient présentant une GNFNA suggère l'existence d'un précurseur au niveau du sérum qui mènerait à la formation de dépôts fibrillaires et expliquerait les récidives après transplantation rénale $[3,4]$.
La GNFNA touche essentiellement les adultes avec une moyenne d'âge de 55-60 ans, une légère prépondérance féminine (ratio : 1,5 femme/1 homme) et une prédilection pour les Caucasiens $[1,4,10]$.

Cliniquement, l'atteinte rénale se manifeste par une protéinurie, néphrotique dans $50 \%$ des cas qui peut être associée à une hématurie micro ou macroscopique ( $60 \%$ des cas), une hypertension artérielle (77\% des cas) et une insuffisance rénale ( $72 \%$ des cas avec une créatininémie $>3,1 \mathrm{mg} / \mathrm{dL}$ au moment du diagnostic) $[1,10]$. La médiane de survie rénale est de 24 mois après le diagnostic anatomopathologique [10]. Une glomérulonéphrite rapidement progressive est cliniquement présente dans un tiers des cas [4]. Il existe une corrélation entre le tableau histologique retrouvé sur la biopsie de départ et l'évolution clinique du patient en termes de sévérité de la protéinurie et d'évolution de la fonction rénale. Ainsi, les patients avec des lésions de glomérulosclérose diffuse ( $>70 \%$ des glomérules) et ceux avec une prolifération diffuse (glomérulonéphrite membranoproliférative et prolifération diffuse avec prolifération endocapillaire ou extracapillaire) évoluent rapidement vers une insuffisance rénale terminale, contrairement aux patients avec une prolifération ou une sclérose mésangiale ou un tableau de glomérulonéphrite extramembraneuse [10].

Différents traitements par corticothérapie et cytotoxiques ont été tentés mais aucun ne semble réellement efficace $[1,10,11]$. Rosensock et al. ont cependant rapporté quelques cas de rémission transitoire sous cyclophosphamide ou ciclosporine [10].

Après transplantation rénale, la récidive au niveau du greffon est fréquente (>50\%). Sur la quinzaine de cas décrits dans la littérature, il semble cependant que l'évolution clinique soit moins péjorative que sur les reins natifs [4,15-18]. 


\section{Conclusions}

Ce cas clinique révèle à nouveau l'importance de la microscopie électronique dans le diagnostic des syndromes néphrotiques. La GNFNA est en effet, une cause rare de syndrome néphrotique (0,5 à $1 \%$ des biopsies de reins natifs) pouvant recouvrir différents tableaux anatomopathologiques en microscopie optique. La microscopie électronique est donc essentielle à la confirmation du diagnostic, mettant en évidence des microfibrilles, organisées de manière anarchique, ressemblant aux fibrilles d'amyloïdes mais plus épaisses que celles- ci.

Dans le cadre du diagnostic différentiel des glomérulopathies à dépôts organisés, il est cependant conseillé de tenir compte également des données d'immunohistochimie et de la coloration rouge Congo pour différencier la GNFNA des autres entités de ce groupe et notamment, de l'amyloïdose.

L'étiologie des GNFNA reste inconnue. Dans le cas présent, il faut, cependant, noter la coexistence de trois pathologies "dysimmunitaires » (GNFNA, RCUH, GIST) sans qu'aucune relation n’ait été mise en évidence entre les trois entités.

\section{Déclaration d'intérêts}

Les auteurs déclarent ne pas avoir de conflits d'intérêts en relation avec cet article.

\section{Références}

[1] Ivanyi B, Degrell P. Fibrillary glomerulonephritis and immunotactoid glomerulopathy. Nephrol Dial Transplant 2004;19(9):2166-70.

[2] Schwartz MM, Korbet SM, Lewis EJ. Immunotactoid glomerulopathy. J Am Soc Nephrol 2002;13(5):1390-7.

[3] Ronco PM, Aucouturier P, Moulin B. Renal Amyloidosis and Glomerular Diseases with Monoclonal Immunoglobulin Deposition. In: Johnson RJ, Feehally J, editors. Comprehensive Clinical Nephrology. first ed., London: Mosby; 2000. p. $11-4$.

[4] Fogo A, Kashgarian M. Diagnostic Atlas Of Renal Pathology, 1st ed., London: Elsevier Saunders; 2005.

[5] Fogo A, Qureshi N, Horn RG. Morphologic and clinical features of fibrillary glomerulonephritis versus immunotactoid glomerulopathy. Am J Kidney Dis 1993;22(3):367-77.

[6] Alpers CE. Immunotactoid (microtubular) glomerulopathy: an entity distinct from fibrillary glomerulonephritis? Am J Kidney Dis 1992;19(2):185-91.

[7] Korbet SM, Schwartz MM, Lewis EJ. The fibrillary glomerulopathies. Am J Kidney Dis 1994;23(5):751-65.

[8] Touchard G, Bauwens M, Goujon JM, Aucouturier P, Patte D. Preud'homme JL. Glomerulonephritis with organized microtubular monoclonal immunoglobulin deposits. Adv Nephrol Necker Hosp 1994;23:149-75.

[9] Schwartz MM. Glomerular diseases with organized deposits. In: Jennette JC, Olson JL, Schwatz MM, editors. Heptinstall's pathology of the kidney. sixth ed. Philadelphia: Lippincott William \& Wilkins; 2007. p. 911-36.

[10] Rosenstock JL, Markowitz GS, Valeri AM, Sacchi G, Appel GB, D'Agati VD. Fibrillary and immunotactoid glomerulonephritis: distinct entities with different clinical and pathologic features. Kidney Int 2003;63(4): 1450-61.

[11] Alpers CE, Kowalewska J. Fibrillary glomerulonephritis and immunotactoid glomerulopathy. J Am Soc Nephrol 2008;19(1):34-7.

[12] Markowitz GS, Cheng JT, Colvin RB, Trebbin WM, D’Agati VD, Hepatitis C. viral infection is associated with fibrillary glomerulonephritis and immunotactoid glomerulopathy. J Am Soc Nephrol 1998;9(12):2244-52.

[13] Hvala A, Ferluga D, Vizjak A, Koselj-Kajtna M. Fibrillary noncongophilic renal and extrarenal deposits: a report on 10 cases. Ultrastruct Pathol 2003;27(5): 341-7.

[14] Masson RG, Rennke HG, Gottlieb MN. Pulmonary hemorrhage in a patient with fibrillary glomerulonephritis. N Engl J Med 1992 Jan 2;326(1):36-9.

[15] Samaniego M, Nadasdy GM, Laszik Z, Nadasdy T. Outcome of renal transplantation in fibrillary glomerulonephritis. Clin Nephrol 2001;55(2):159-66.

[16] Pronovost PH, Brady HR, Gunning ME, Espinoza O, Rennke HG. Clinical features, predictors of disease progression and results of renal transplantation in fibrillary/immunotactoid glomerulopathy. Nephrol Dial Transplant 1996 11(5):837-42.

[17] Korbet SM, Rosenberg BF, Schwartz MM, Lewis EJ. Course of renal transplantation in immunotactoid glomerulopathy. Am J Med 1990;89(1):91-5.

[18] Carles X, Rostaing L, Modesto A, Orfila C, Cisterne JM, Delisle MB, et al. Successful treatment of recurrence of immunotactoid glomerulopathy in a kidney allograft recipient. Nephrol Dial Transplant 2000;15(6):897900. 